\title{
RETRACTED ARTICLE: Prognostic Significance of Osteopontin and Carbonic Anhydrase 9 in Human Brain Tumors: A Meta-Analysis
}

\author{
Liang-Yu Chen • Li-Bo Liu • Xin-Xing Li • Qi Yu • Bo Yu • \\ Yang Hong • Jian Zheng • Yi-Xue Xue • Yun-Hui Liu
}

Received: 18 August 2014 / Accepted: 24 September 2014 / Published online: 17 August 2015

(C) Springer Science+Business Media New York 2014

The Publisher and Editor retract this article in accordance with the recommendations of the Committee on Publication Ethics (COPE). After a thorough investigation we have strong reason to believe that the peer review process was compromised.

The original article was published online on October 23, 2014.

L.-Y. Chen · X.-X. Li • Q. Yu • B. Yu • Y. Hong · J. Zheng •

Y.-H. Liu $(\bowtie)$

Department of Neurosurgery, Shengjing Hospital of China Medical

University, Sanhao Street No.36, Shenyang, Liaoning

Province 110004, People's Republic of China

e-mail: liuyh@sj-hospital.org

L.-B. Liu • Y.-X. Xue

Department of Neurobiology, College of Basic Medicine, Shenyang,

Liaoning Province 110004, People's Republic of China

L.-B. Liu • Y.-X. Xue

Institute of Pathology and Pathophysiology, China Medical

University, Shenyang, Liaoning Province 110004,

People's Republic of China 